\title{
Multiple effectiveness aspects of tapentadol for moderate-severe cancer-pain treatment: an observational prospective study
}

This article was published in the following Dove Medical Press journal: Journal of Pain Research

\section{Cascella' \\ CA Forte' \\ S Bimonte' \\ G Esposito' \\ C Romano ${ }^{2}$ \\ R Costanzo ${ }^{2}$ \\ A Morabito 2 \\ A Cuomo'}

'Department of Anesthesia and Pain Medicine, Istituto Nazionale Tumori, IRCCS - Fondazione G Pascale, Naples, Italy; ${ }^{2}$ Thoracic Medical Oncology, Istituto Nazionale Tumori, IRCCS - Fondazione G Pascale, Naples, Italy
Correspondence: S Bimonte Department of Anesthesia and Pain Medicine, Istituto Nazionale Tumori, IRCCS - Fondazione G Pascale, Naples, Italy

Tel +39 08I 590322 I

Email s.bimonte@istitutotumori.na.it
Background: Previous studies have shown the efficacy of tapentadol (TP) for chronic cancer pain. We evaluated multiple effectiveness aspects of TP prolonged release on moderate-severe cancer-related pain, neuropathic pain $(\mathrm{NeP})$, patient satisfaction, and quality of life.

Methods: An observational prospective study was conducted on 80 cancer patients. Opioidnaïve patients received a starting dose of prolonged-release TP $50 \mathrm{mg}$ twice daily, and opioidexperienced patients were switched to TP, not to exceed $500 \mathrm{mg} /$ day. Treatment response was evaluated at 3, 6, 30-40, and 60-70 days through response rate, numeric rating-scale scoring, survival analysis (time to event for response), pain-intensity difference, TP escalation-index percentage, and effects on NeP. The drug-sparing effect on concomitant therapies was evaluated. Results: Seventy of 80 patients ( $88 \%$ ) were responders to treatment (95\% CI 78\%-94\%). Compared to $\mathrm{T}_{0}$, pain-intensity reductions were statistically significant for all intervals $(P<0.01)$, with better results at $\mathrm{T}_{3} / \mathrm{T}_{4}$. NeP was significantly reduced at $\mathrm{T}_{4}(P<0.01)$. The probability of response was low at the initial stages and increased during the study. Pain-intensity differences decreased during the study, though without significance. Two patients $(2.5 \%)$ left the study for TP-induced side effects. A significant improvement in quality of life was observed after 30-40 days $(P<0.01)$. The majority of patients were "satisfied", "very satisfied", or "extremely satisfied" $\left(\mathrm{T}_{3}-\mathrm{T}_{4}\right)$.

Conclusion: TP was effective in terms of drug-sparing effect, response rate, TP escalationindex percentage, and $\mathrm{NeP}$ management. By comparing data from the survival analysis with the response rate and time to response (numeric rating scale from $\mathrm{T}_{0}$ to $\mathrm{T}_{4}$ ), we found that although TP induced a quick response, a longer period of therapy and higher doses were needed to improve the positive result.

Keywords: pain, cancer pain, tapentadol, quality of life, patient satisfaction, neuropathic pain

\section{Introduction}

Cancer-pain management remains a significant challenge in medicine. Recent metaanalytic findings demonstrated that cancer pain affected about $55 \%$ of patients in the course of therapy, and approximately two-thirds of those in terminal-disease stages. Furthermore, moderate-severe pain was found in $38 \%$ of patients. ${ }^{1}$ Due to the fact that in a high percentage of cases, malignancy-associated pain negatively affects functional status and quality of life (QOL), it represents one of the most disabling symptoms in cancer patients, ${ }^{2}$ prejudicing patients' well-being on multiple levels, even when of mild intensity. ${ }^{3}$ According to guidelines from different scientific societies, opioids are the cornerstone of cancer-pain therapy. ${ }^{4-6}$ Nevertheless, by using opioids, clinicians must often face problems related to efficacy and tolerability, and these obstacles often lead to poor compliance or withdrawal from treatment. ${ }^{7}$ For instance, approximately $40 \%$ 
of cancer patients experience opioid-induced nausea and vomit, and up to $95 \%$ report opioid-induced constipation. ${ }^{8}$ Consequently, research on effective and well-tolerated pain therapies represents a field of study with clinical impact of huge importance. Toward this objective, an effective strategy could be the use of molecules acting by combining more than one mechanism of action.

Tapentadol (TP) is an opioid with a central mechanism of action that combines $\mu$-opioid-receptor (MOR) agonism with noradrenaline-reuptake inhibition (NRI). ${ }^{9}$ Probably, the moderate affinity on MOR combined with the opioid-sparing effect due to NRI is responsible for the well-tolerable profile of TP. ${ }^{10}$ In chronic cancer and noncancer pain patients, Meng et al's meta-analysis showed that the use of TP was associated with a better tolerability profile - lower withdrawal rate and adverse events (AEs)/constipation — when compared with other opioids. ${ }^{11}$ In a prospective study on opioid-naïve cancer patients, Mercadante et $\mathrm{al}^{12}$ demonstrated that TP was effective and well tolerated for treating moderate-severe chronic pain. Furthermore, in a randomized controlled trial carried out on patients with moderate-severe chronic cancer pain, Kress et $\mathrm{al}^{13}$ found that the response rate was significantly higher in the TP group compared with placebo. Moreover, during titration TP showed comparable efficacy to morphine, with reduced occurrence of gastrointestinal side effects. In another randomized controlled trial, Imanaka et $\mathrm{al}^{14} \mathrm{dem}-$ onstrated that TP had efficacy not inferior to oxycodone. The same authors conducted a subsequent investigation in patients successfully treated with opioids, and found maintenance of efficacy after rotation to TP from another strong opioid. ${ }^{15}$ Other studies have proved that TP prolonged-release
(PR) reduces pain-related mental and physical burden and improved QOL. ${ }^{16}$ Again, a recent Cochrane systematic review showed that TP was similar to oxycodone or morphine in terms of efficacy and tolerability, although the authors found insufficient findings to obtain pooling of data. Therefore, they concluded that for better efficacy assessment, further clinical investigations should focus on more precise end points, such as number of responder participants and detailed efficacy indices. ${ }^{17}$

This study aimed to investigate multiple effectiveness aspects of TP PR when used as routine clinical practice on moderate-severe chronic cancer-related pain. Effects on the neuropathic component of the pain, QOL, and patient satisfaction (PS) were also investigated. A careful tolerability analysis was out of the scope of this study, as a detailed picture of TP side effects had been offered by previous controlled $^{13-15}$ and uncontrolled ${ }^{12,16,18}$ investigations. ${ }^{19}$

\section{Methods}

\section{Study population and design}

This clinical investigation was carried out according to the Declaration of Helsinki's ethical principles. Individuals with a diagnosis of any type of cancer and experiencing chronic moderate-severe (numeric rating scale $[\mathrm{NRS}] \geq 5$ on an 11-point scale) pain on the day of enrollment were included in this prospective cohort study from November 2015 to February 2017. Approval from the Institutional Medical Ethical Committee (protocol 29/15 OSS) of the Istituto Nazionale Tumori - Fondazione Pascale, Naples was obtained, and patients signed an informed consent before enrolling in the study. Inclusion and exclusion criteria are summarized in Table 1.

Table I Inclusion and exclusion criteria

\begin{tabular}{|l|l|}
\hline Inclusion criteria & Exclusion criteria \\
\hline Consenting patients aged $>18$ years & Unable to provide informed consent \\
Life expectancy $>3$ months & Drug abuse/alcoholism \\
Pain intensity: NRS $\geq 5$ & Renal $\left(\mathrm{GFR}<30 \mathrm{~mL} / \mathrm{min} / \mathrm{I} .73 \mathrm{~m}^{2}\right)$ or liver impairment* \\
No severe cognitive impairment & No malignant chronic pain $\mathrm{syndromes}^{*}$ \\
Male/female patients & Severe respiratory failure $\left(\mathrm{PaO}_{2}<50 \mathrm{mmHg}\right)$, untreated asthma, OSA \\
Opioid-naïve or opioid-experienced & Acute pancreatitis or biliary-tract disease \\
& Paralytic ileus \\
& IBD \\
& NYHA $>3$ \\
& Recent ACS (6 months) \\
& Recent stroke, head injury (I year) \\
& Medication: MAOI \\
& Nursing mothers \\
& Lactose intolerance \\
\hline
\end{tabular}

Notes: *ALAT >45 U/L; ASAT > 35 U/L; ALP > 05 U/L; GT >45 U/L (age 18-39 years) or GT>75 U/L (age over 39 years).

Abbreviations: ACS, acute coronary syndrome; GFR, glomerular filtration rate; IBD, inflammatory bowel disease; MAOI, monoamine oxidase inhibitors; NRS, numeric rating scale; NYHA, New York Heart Association; OSA, obstructive sleep apnea. 
TP PR was administered following the summary of product characteristics. ${ }^{20}$ Opioid-naïve patients received a starting dose of TP PR $50 \mathrm{mg}$ twice daily, and opioid-tolerant individuals were switched to TP in an equianalgesic condition (1:3.3 ratio with oral morphine equivalents $)^{21}$ not to exceed $500 \mathrm{mg} /$ day. Both opioid-naive and opioid-tolerant patients patients were being allowed to take opioids to treat breakthrough cancer pain. Similarly, the use of other medications (eg, adjuvants or drugs for concomitant diseases) was allowed, unless they were indicated in the exclusion criteria (Table 1). During the study, patients were submitted to five consultations $\left(\mathrm{T}_{0}-\mathrm{T}_{4}\right)$ : baseline examination $\left(\mathrm{T}_{0}\right)$ for enrollment and data collection, including demographic data, tumor type, performance status (Eastern Cooperative Oncology Group [ECOG] 0-5 scale), ${ }^{22}$ and after 3 days $\left(\mathrm{T}_{1}\right), 6$ days $\left(\mathrm{T}_{2}\right), 30-40$ days $\left(\mathrm{T}_{3}\right)$, and 60-70 days $\left(\mathrm{T}_{4}\right)$.

\section{Study end points and assessments}

The primary end point was evaluation of treatment response. A responder patient was defined as one who achieved and maintained a clinical result $(\mathrm{NRS} \leq 3)$ for the whole duration of the study. A positive result was indicated as the goal of $75 \%\left(\pi_{1}\right)$ of response rate, with at least $60 \%\left(\pi_{0}\right)$ of responder patients. Consequently, the null hypothesis was $\pi_{0}=\pi_{1}$, whereas the alternative hypothesis (HA) was $\pi_{0}<\pi_{1}$. The survival analysis (ie, analysis of time-to-event data) has been used for evaluating the time needed to obtain the treatment response.

The efficacy of the TP treatment was investigated by:

- recording the pain intensity (NRS) in the different phases of the study and analysis of pain-intensity difference (PID), which represents the difference in NRS values between any observation time and the baseline;

- survival analysis (time-to-event [treatment response] data);

- the neuropathic component (NeP) of patients' cancer pain through the Douleur Neuropathique 4 (DN4) score; ${ }^{23}$

- the TP-dosage trend;

- the TP escalation-index percentage (TPEI\%).

According to Mercadante et al, ${ }^{24}$ the TPEI correlates the mean percentage dosage of a drug from the starting dose (TPSD). With maximal TP dosage (TPMD) obtained, the formula was ([TPMD - TPSD]/TPSD)/days $\times 100$.

Secondary end points were:

- QOL evaluation, conducted through Spitzer's QOL index (QOL Index), ${ }^{25}$ scored at baseline and compared with values recorded at the other study intervals;
- PS investigation, wherein we asked patients ( at $\mathrm{T}_{3}$ and $\mathrm{T}_{4}$ ) to indicate their level of satisfaction from one of "very dissatisfied", "dissatisfied", "indifferent", "satisfied", "very satisfied", or "extremely satisfied";

- drug-sparing effect on concomitant therapies (ie, analgesics, adjuvants);

- treatment-related AEs, recorded though not submitted for statistical analysis. (descriptive analysis performed for AEs inducing dropouts).

\section{Statistical analyses}

The first evaluation of the main variable (ie, rate of responder patients) was analyzed by the exact binomial test ( $\left.\varpi_{0} 60 \%\right)$, evaluating the number of responders with respect to the number of patients enrolled. Percentage and confidence interval (CI) 95\% were also reported. The other evaluations of the main variable (ie, Responder Yes/No) were studied by the logistic regression analysis (age, sex, ECOG score, baseline NRS were used as covariates) and through survival analysis using the Kaplan-Meier method.

Data on NRS and QOL were assessed by ANOVA for repeated measures without grouping, with multiple comparisons vs baseline. The sample of patients with NeP (DN4) was instead evaluated by the McNemar test. Multiple comparisons were made vs baseline detection. PID, number of dropouts, dose-increase index, and AEs are reported in a descriptive manner. The 9.4 version of SAS was adopted for data analysis.

\section{Results}

Table 2 summarizes demographic and clinical data for the population under study. Among the 80 patients included, the most represented age-group was 60-70 years (27 cases, $34 \%$ ). Approximately $50 \%$ of patients were older than 64 years. Most patients were relapsed or in disease progression $(n=38)$, whereas only in $11 \%$ of cases was cancer at an early clinical stage. A total of 34 patients (42\%) had an ECOG value of 2 (Ambulatory and capable of all self-care, but unable to carry out any work), and in 34 cases (42\%) no anticancer therapeutic approaches were ongoing at the start of the study. The mean duration of painful conditions was 4.4 months, and no patients had pain duration $>1$ year. We included 76 opioid-naïve patients (95\%) and four opioidexperienced individuals (5\%).

\section{Primary end point (efficacy)}

Within the first 60 days of TP treatment, 70 of 80 patients $(88 \%)$ were responders to treatment (95\% CI 78\%-94\%). The 
Table 2 Baseline demographic and clinical data $(n=80)$

\begin{tabular}{|c|c|}
\hline \multicolumn{2}{|l|}{ Sex, n (\%) } \\
\hline Male & $37(46.2)$ \\
\hline Female & $43(53.8)$ \\
\hline Age (mean \pm SD) & $63.3 \pm 12.5$ \\
\hline Weight* & $72.8 \pm 11.8$ \\
\hline \multicolumn{2}{|l|}{ Tumor type, n (\%) } \\
\hline Lung & $15(18.8)$ \\
\hline Breast & $14(17.5)$ \\
\hline Gastrointestinal & $23(28.8)$ \\
\hline Urological & $10(12.5)$ \\
\hline Head/neck & $4(5)$ \\
\hline Gynecological & $2(2.5)$ \\
\hline Others $\#$ & $12(15)$ \\
\hline \multicolumn{2}{|l|}{ Stage of disease, $\mathbf{n}(\%)$} \\
\hline Initial & $9(11.2)$ \\
\hline Under treatment & $33(41.2)$ \\
\hline Relapsed/progression & $38(47.6)$ \\
\hline \multicolumn{2}{|l|}{ Previous analgesic therapy (n/\%) } \\
\hline Opioid-naïve & $76(95)$ \\
\hline Opioid-experienced & $4(5)$ \\
\hline Other analgesic $\varsigma^{\S}$ & $20(25)$ \\
\hline \multicolumn{2}{|l|}{ ECOG performance status (n/\%) } \\
\hline 0 & $\mathrm{I}(\mathrm{l} .2)$ \\
\hline 1 & $17(2 \mid .2)$ \\
\hline 2 & $34(42.5)$ \\
\hline 3 & $23(28.8)$ \\
\hline 4 & 0 \\
\hline 5 & 0 \\
\hline Not available & $5(6.2)$ \\
\hline \multicolumn{2}{|c|}{ Concomitant anticancer therapy, n (\%) } \\
\hline Chemotherapy & $30(37.5)$ \\
\hline Radiotherapy & $14(17.5)$ \\
\hline Others & $2(2.5)$ \\
\hline No therapy & $34(42.5)$ \\
\hline Comorbidities, n (\%) & $16(20)$ \\
\hline Previous surgery, n (\%) & II (13.8) \\
\hline Duration of pain^ (mean \pm SD) & $4.4 \pm 3.2$ \\
\hline
\end{tabular}

Note: *Data not available for seven patients; ${ }^{*}$ melanoma (2), pancreas (I), bone (2), hematologic (2), not specified (5); ^expressed in months; ${ }^{5}$ NSAIDs $12.5 \%$, acetaminophen $8.8 \%$, others $3.8 \%$; ' ${ }^{\text {c }}$ ardiovascular $6.2 \%$, hepatic $1.2 \%$, renal $3.8 \%$, others $8.8 \%$.

Abbreviations: ECOG, Eastern Cooperative Oncology Group; NSAIDs, nonsteroidal anti-inflammatory drugs.

percentage of responders was significantly higher $(P<0.01)$ at the value of $60 \%$, corresponding to the minimum percentage to consider the effect of treatment to be clinically relevant (HA satisfied; Figure 1).

In the regression analysis, no covariate investigated (ie, age, sex, ECOG scores, or baseline NRS values) was statistically significant at the 0.01 level (Figure 2).

The survival analysis (70 responders) showed that the probability of response was low at the initial stages and increased during the study: it was about $3 \%$ after 3 days, approximately $23 \%$ at 35 days, and $>90 \%$ at 65 days (Figure 3 ).
Analysis of the NRS results demonstrated that in the transition from $\mathrm{T}_{0}$ to $\mathrm{T}_{1}$, the intensity of pain decreased by $11 \%$ (from an average value of 7.1 to 6.3). Subsequently, this trend was confirmed, as compared to baseline the reduction was $15 \%$ (NRS 6.0) at $\mathrm{T}_{2}, 38 \%$ (NRS 4.4) at $\mathrm{T}_{3}$, and $59 \%$ (NRS 2.9 ) at the final consultation. Compared to $T_{0}$, pain-intensity reductions were statistically significant for all evaluations $(P<0.01$; Figure 4).

In the PID analysis, at the first evaluation the average difference in NRS was 0.8 points. During the study, PID values progressively increased: 1.2 at $\mathrm{T}_{2}, 2.6$ at $\mathrm{T}_{3}$, and finally 4.2 at $\mathrm{T}_{4}$, though never reached significance (Figure 5).

The mean TP dosage increased in the course of the study. It was about $185 \mathrm{mg} /$ day at baseline, $200 \mathrm{mg}$ at $\mathrm{T}_{1}$, and 210 $\mathrm{mg}$ at the end of the first week $\left(\mathrm{T}_{2}\right)$. At $\mathrm{T}_{3}$ and the final evaluation, it reached $301 \mathrm{mg} /$ day and $365 \mathrm{mg} /$ day, respectively (Figure 6).

Mean TPEI was 2.4\%: half the patients were $<1.6 \%$, and in no patient was it $>33.3 \%$. The percentage of patients with $\mathrm{NeP}$ diagnosis progressively decreased during the study. Decreases were from $59 \%\left(\mathrm{~T}_{0}\right)$ to $50 \%\left(\mathrm{~T}_{3}\right)$ and to $45 \%\left(\mathrm{~T}_{4}\right)$. $\mathrm{T}_{1} / \mathrm{T}_{2}$ were not considered, as they were too close to baseline examination. At the last evaluation compared to baseline, the difference was significant $(P<0.01$; Figure 7$)$.

\section{Secondary end points (well-being/ tolerability)}

From an average value of $4.8 / 10$ at baseline $\left(\mathrm{T}_{0}\right)$, average QOL results at $\mathrm{T}_{1}$ and $\mathrm{T}_{2}$ were of $4.9 / 10(+3 \%)$ and 5.0/10 $(+5 \%)$, respectively. Scores improved by $21 \%$ from study enrolment to evaluation at 30-40 days (5.8/10), and subsequent $\left(\mathrm{T}_{4}\right)$ values remained substantially unaltered $(5.9 / 10$, $23 \%$ increment). For both last evaluations, compared to the beginning of the study, the increase was statistically significant $(P<0.01$; Figure 8$)$.

The majority of patients were "satisfied", "very satisfied", or "extremely satisfied" at both $\mathrm{T}_{3}$ and $\mathrm{T}_{4}$ follow-up $\left(\mathrm{n}=68[85 \%]\right.$ at $\mathrm{T}_{3}, \mathrm{n}=67$ [84\%] at $\left.\mathrm{T}_{4}\right)$. Three patients $(4 \%)$ at $\mathrm{T}_{3}$ and two $(2.5 \%)$ at $\mathrm{T}_{4}$ were "very dissatisfied" or "dissatisfied". The proportion of patients experiencing AEs that caused withdrawal from the study was $2.5 \%$ (two patients). One patient experienced nausea and vomiting, and the other severe opioid-induced constipation. The other four patients were considered dropouts (total $n=6$ ) due to not being able to complete the study (exit [n=1], not completing follow-up $[\mathrm{n}=3])$. Among the concomitant pharmacological therapies, during the course of the study we found reduced use of drugs as prescription of the anticonvulsant gabapentin progressively 
Primary endpoint

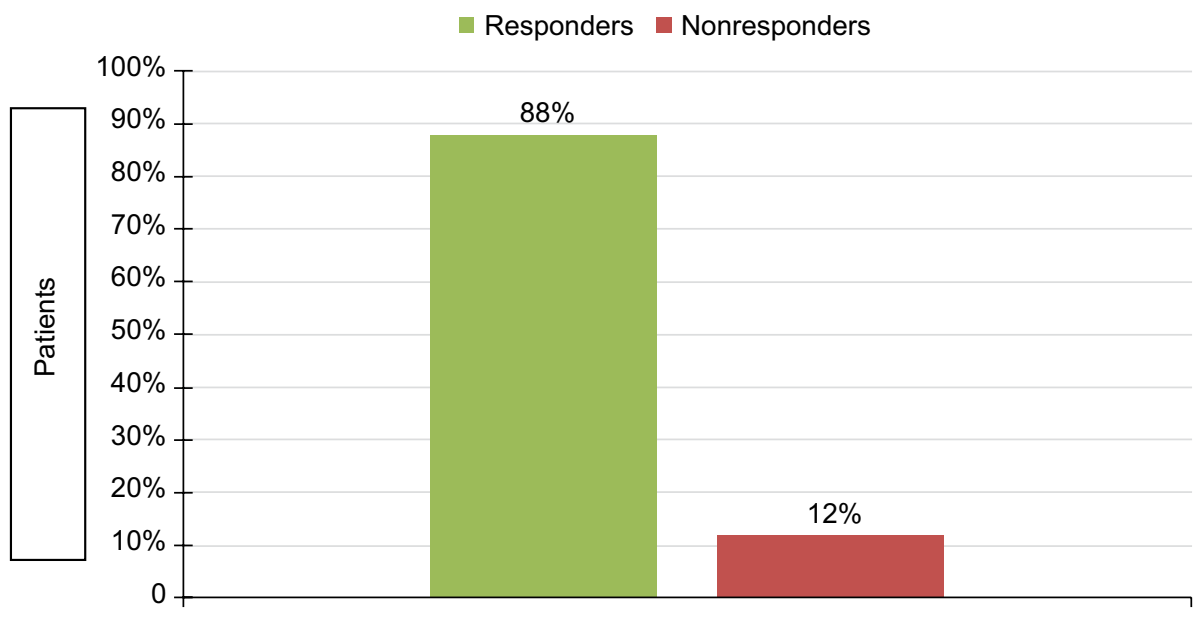

Figure I Primary end point.

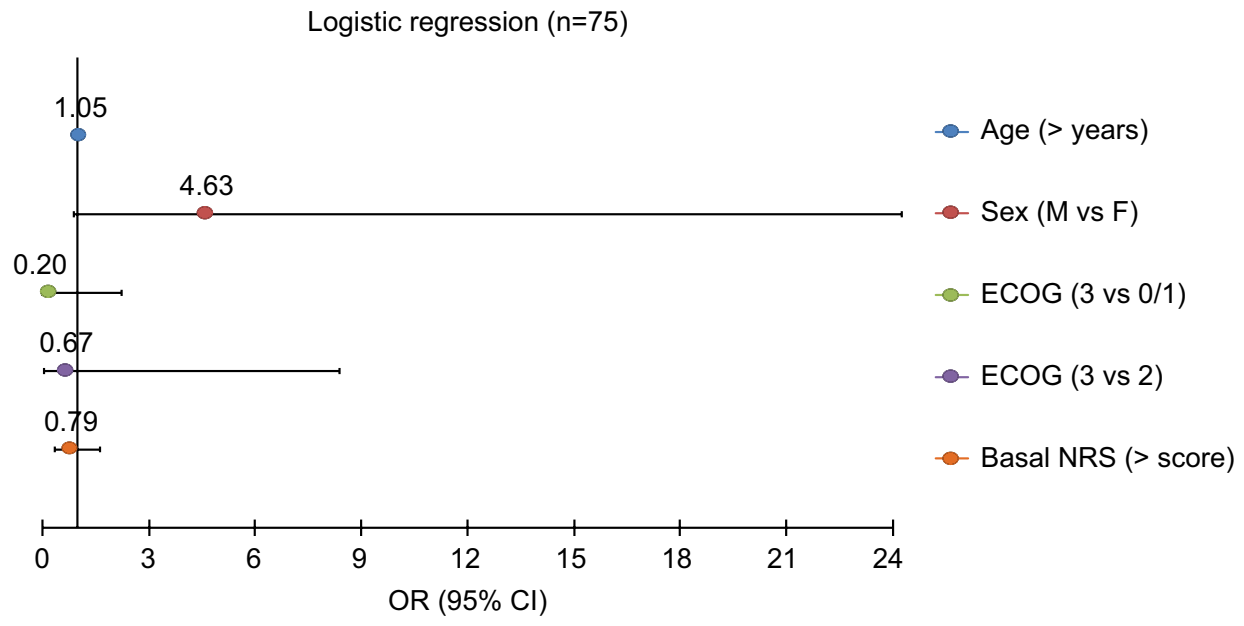

Figure 2 Regression analysis showed that no covariate tested was significant.

Abbreviations: ECOG, Eastern Cooperative Oncology Group; NRS, numeric rating scale.

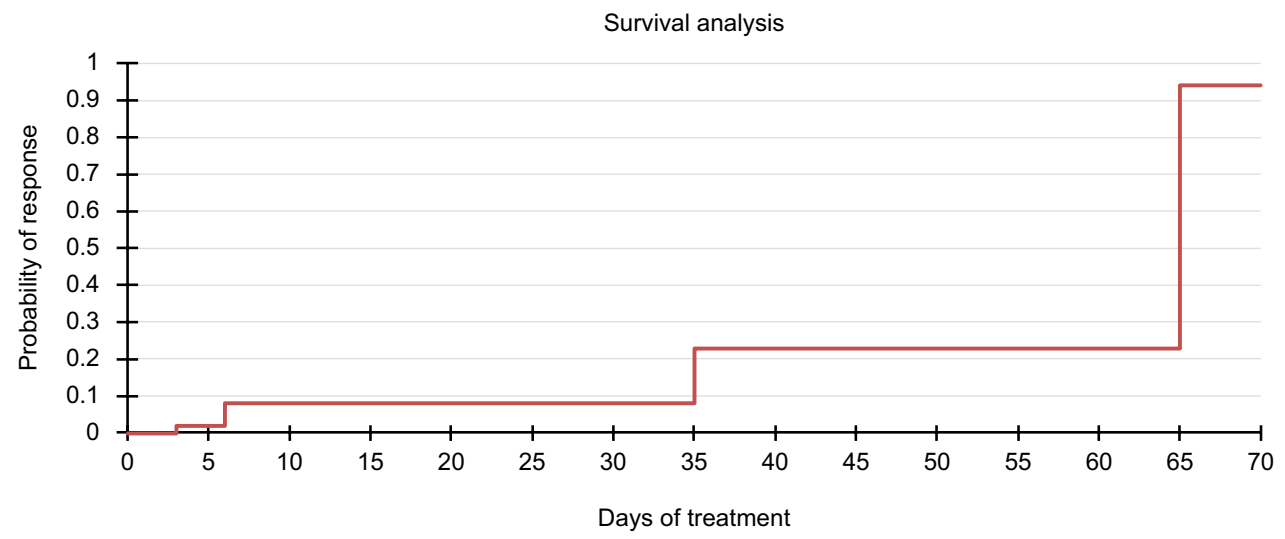

Figure 3 Survival analysis: median time to response 65 days. 


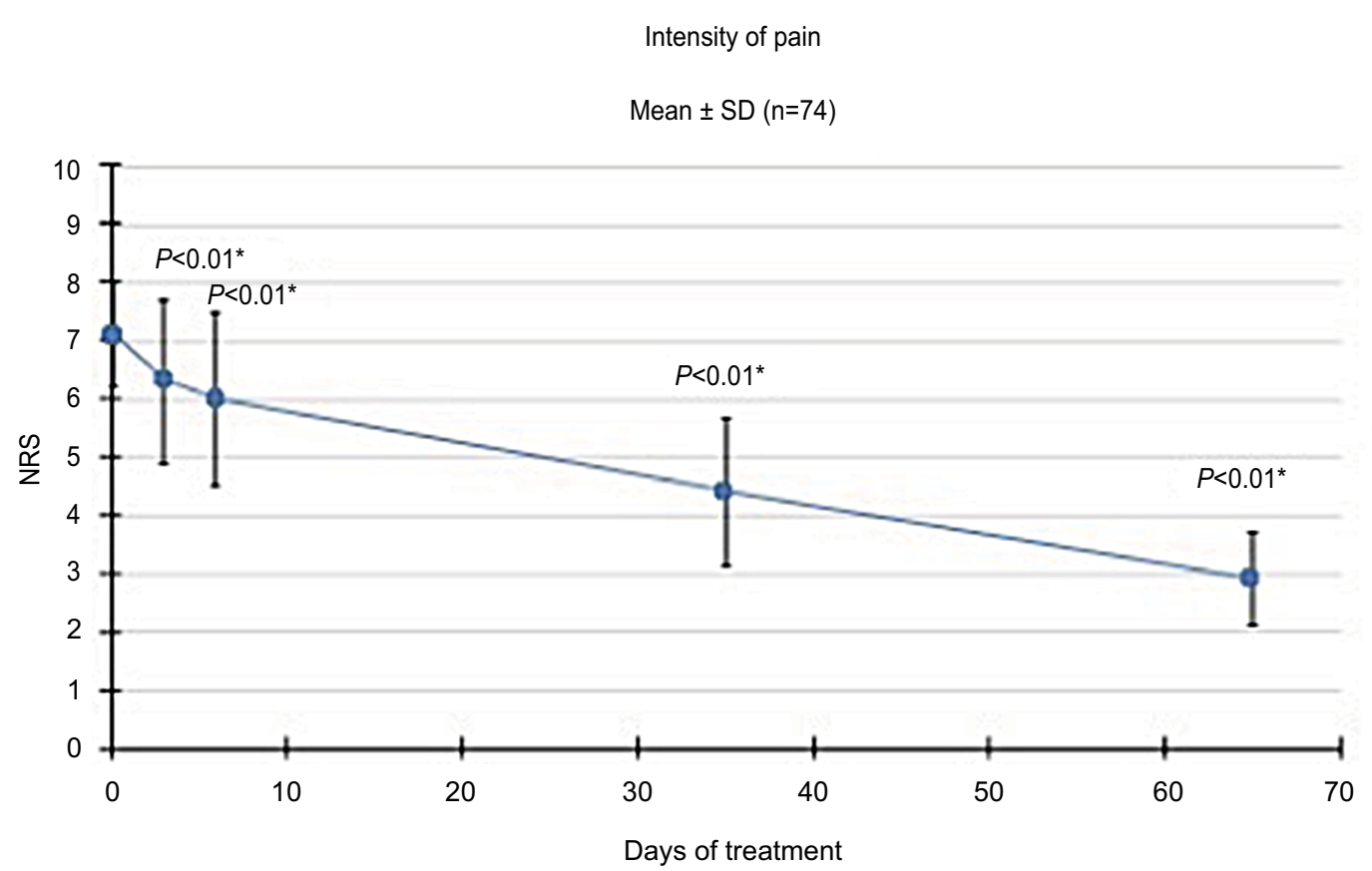

Figure 4 Pain intensity.

Notes: Pain-intensity reductions were statistically significant compared to $T_{0}$ for all evaluations $(* P<0.0 I)$.

Abbreviation: NRS, numeric rating scale.

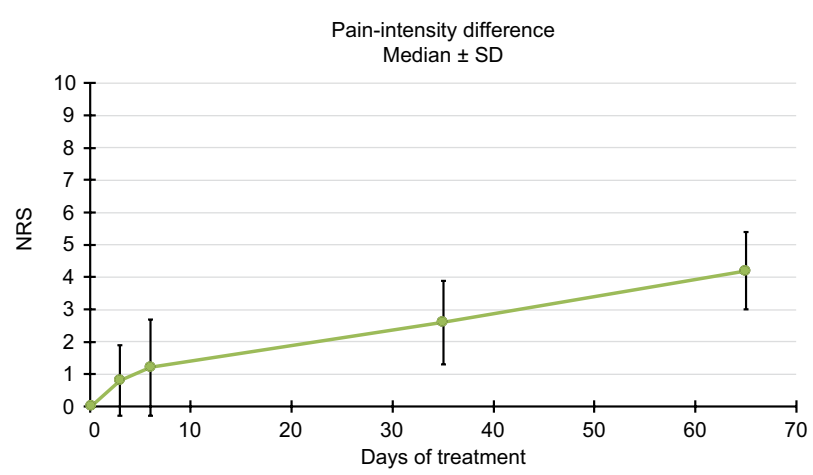

Figure 5 Pain-intensity differences.

Note: During the study, pain-intensity difference progressively increased, although never reached significance.

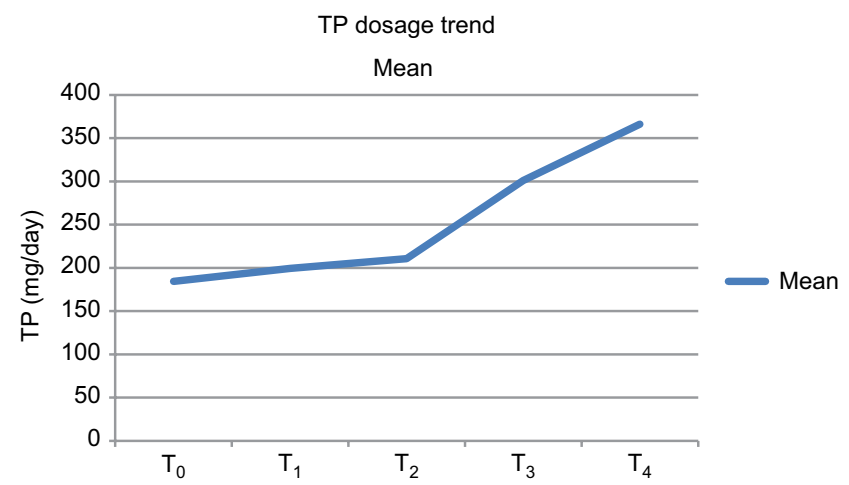

Figure 6 TP-dosage trend.

Note: Mean TP dosage increased in the course of the study. SD: $89.0\left(T_{0}\right), 81.6\left(T_{1}\right)$, $86.0\left(T_{2}\right)$, II3.I $\left(T_{3}\right)$, I37.I $\left(T_{4}\right)$.

Abbreviation: TP, tapentadol. decreased from $62 \%$ of patients $\left(\mathrm{T}_{0}-\mathrm{T}_{1}\right)$ to $55 \%$ (last evaluation) and acetaminophen from $61 \%$ to $48 \%$.

\section{Discussion}

This study evaluated several effectiveness aspects of analgesic therapy in cancer patients who experienced moderatesevere pain and received TP for pain relief. Because this was a purely observational study, the inclusion of the cutoff was aimed at offering data from a statistical approach and not to draw definitive conclusions. On these bases, the statistical analysis indicated that the established goal of $75 \%$ of the responder rate was exceeded (88\%). Consequently, the HA was satisfied, and in turn the primary end point indicated an improvement in pain control. At the beginning of the study (transition from $\mathrm{T}_{0}$ to $\mathrm{T}_{1}$ ), the intensity of pain decreased by $11 \%$, and subsequently this trend was confirmed, because compared to baseline, NRS reduction was up to $15 \%$ at the end of the first week of TP administration $\left(\mathrm{T}_{2}\right)$. We found that pain intensity significantly decreased after the first month of treatment and adequate pain relief maintained during the entire study period. Compared to $\mathrm{T}_{0}$, PID was statistically significant for all evaluations $(P<0.01)$. In order to find variables associated with the opioid response, though no covariate tested was significant, the response rate increased with age and was higher in women than men.

In regard to NeP, DN4 scores were significantly reduced at the end of treatment. This finding is of paramount impor- 
DN4 $(\mathrm{N}=48)$

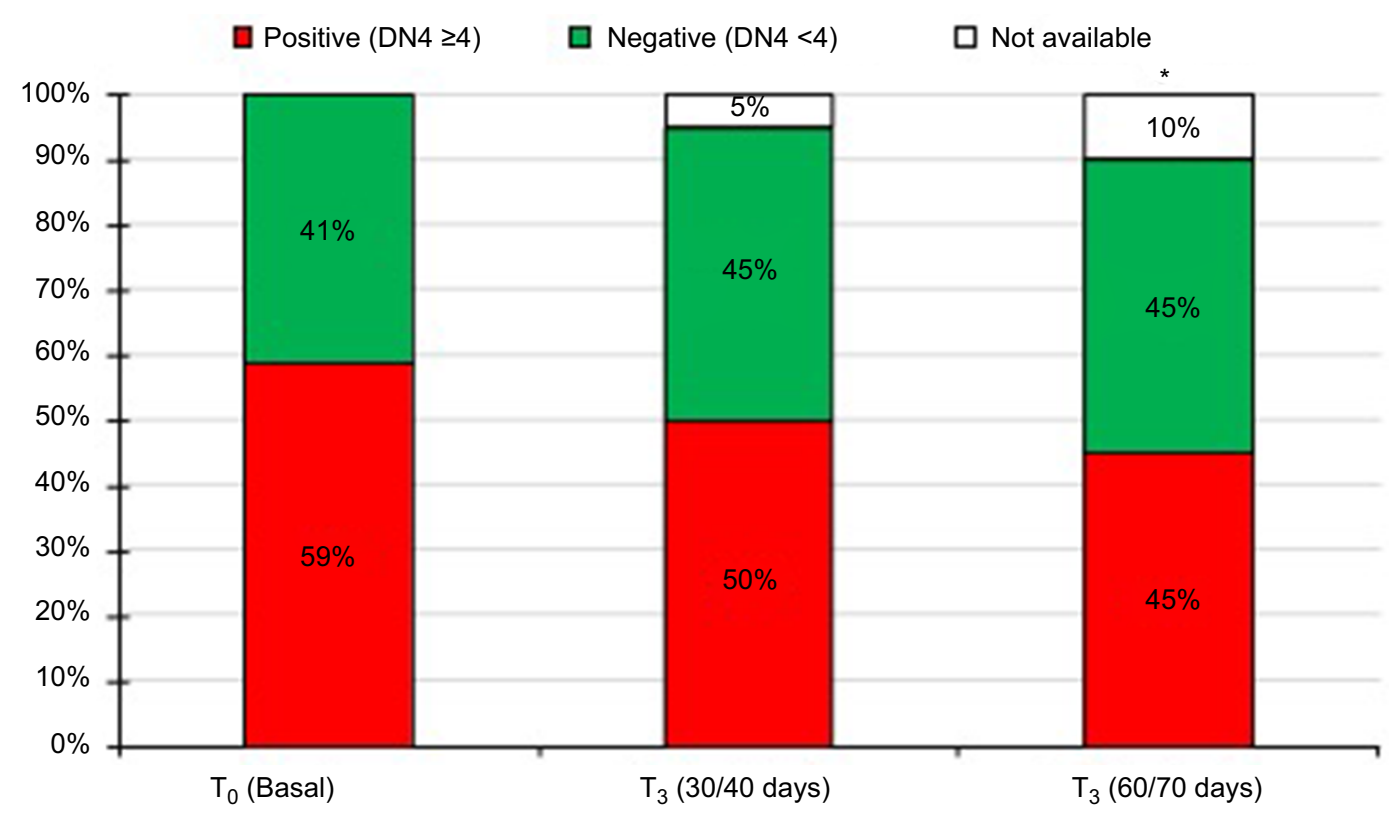

Figure $7 \mathrm{NeP}$ analysis.

Notes: The percentage of patients with NeP progressively decreased during the study. At the last evaluation compared to baseline, the difference was significant ( $* P<0.0 \mathrm{I}$ ). Abbreviations: DN, Douleur Neuropathique; NeP, neuropathic pain.

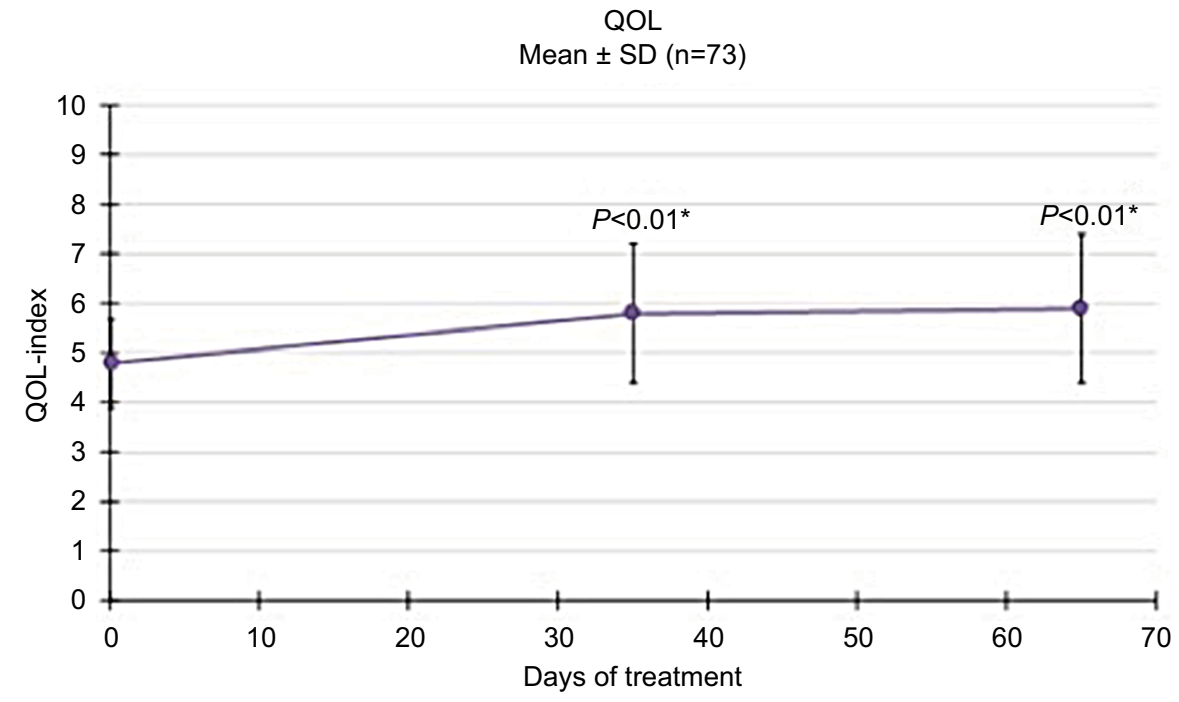

Figure 8 Evaluation of quality of life (QOL).

Note: $Q O L$ progressively increased from the beginning of the study $(P<0.0 \mathrm{I})$.

tance, as cancer pain is often a mixed-mechanism pain state with a well-represented neuropathic component. ${ }^{26}$ Several investigations have demonstrated a beneficial effect of TP on $\mathrm{NeP},{ }^{12}$ even in advanced cancer. ${ }^{27}$ This effect can be in part be explained as a consequence of the combined mechanism of MOR and NRI. In vivo studies have dissected TP-induced opioid and noradrenergic actions. ${ }^{28}$ Nevertheless, the precise mechanism underlying the effect of TP on NeP should be better explained. ${ }^{29}$

While the overall withdrawal rate was $7.5 \%$, TP-induced dropouts for AEs were $2.5 \%$. Again, no patient left the study due to uncontrolled pain. This finding correlates with the mean TP dosage and the escalation ratio (TPEI\%). The former increased in the course of the study, although in all cases good pain relief 
was obtained with a dosage of $\leq 500 \mathrm{mg} /$ day (cutoff). Moreover, the mean TPEI\% was very low and never surpassed $33.3 \%$. At the last observation, the mean TP dosage had approximately doubled from baseline (366vs 184.4). These data contrast with results of previous studies, ${ }^{21}$ suggesting lower tolerance at the end of the study. However, the lack of uniformity of the sample cannot allow the drawing of conclusive results.

The survival analysis was conducted by considering response to treatment as an event. This statistical approach is a probability analysis, which is often necessary to overcome dropout bias (ie, censored observation). For each patient, it was considered the first observation time in which an NRS score $\leq 3$ was reached and maintained without interruption. Although we found that the probability of response increased during the study, it was very low at initial stages, whereas the survival time for obtaining the highest probability corresponded with the last control. Further studies are needed to explain these surprising results. Data from other investigations are not available, because in no study on the topic a survival analysis was conducted. By comparing these findings with the response-rate data and the dose analysis, we may deduce that despite TP inducing a quick response, a longer period of therapy and higher doses are necessary to maintain the result.

Overall, the general state of health of the patients improved, despite the underlying illness. Compared with baseline observations, QOL index values had increased at the third interval (30-40 days) in a significant manner, and this result was maintained until the end of the study $\left(\mathrm{T}_{4}\right)$. However, the analgesic therapy did not ameliorate QOL in the initial stages of the investigation $\left(\mathrm{T}_{1} / \mathrm{T}_{2}\right)$. Furthermore, we found a positive association between TP use and PS in the majority of patients. Probably, the good tolerability profile of TP may explain the improved patient compliance. We collected interesting data on the drug-sparing effect of TP. In particular, we observed reduced use of gabapentin and acetaminophen already in the early stages of the study.

\section{Study limitations}

This study presents several limitations, consisting mainly of the uncontrolled design. Another limitation is the heterogeneity of the sample concerning the analgesic regimen at baseline. Moreover, the sample size and heterogeneity of the clinical setting concerning the stage of disease, as well as cancer type and therapies, do not allow us to draw definite conclusions. Again, the study was not segregated by concomitant pharmacological therapies or age-matched controls, which can greatly influence the parameters. As a consequence, our statistical approach (eg, the cutoff) was aimed at offering data to be used in controlled studies and not to draw definitive conclusions. Furthermore, we enrolled both opioid-naïve and opioid-experienced patients. Although several previous investigations have proved the efficacy and tolerability of TP in opioid-naïve patients, ${ }^{12}$ the opioid-switching approach is often a winning strategy, ${ }^{30,31}$ and prospective evaluations focused only on opioid-tolerant patients proved that TP was effective and well tolerated, even when used in doses of 350-450 mg/ day. ${ }^{18}$ In regard to other study limitations, opioids used to manage breakthrough cancer pain and potential concomitant therapies could have influenced the TP effect.

\section{Conclusion}

Despite its limitations, this observational study offers interesting findings on the efficacy of TP on cancer-related pain, especially in terms of response rate, dosages (ie, low TPEI\%), NeP, and drug-sparing effect. The positive effects on pain intensity (ie, NRS reduction) were recorded from the early stages of the study, although the results became clinically relevant after approximately the first month of TP administration. By comparing the data obtained from the survival analysis with response rates, dose analysis, and time to response (NRS from $\mathrm{T}_{0}$ to $\mathrm{T}_{4}$ ), we deduced that despite TP being able to induce a rapid response, probably a longer period of therapy and higher doses are necessary to maintain and improve such a result. This finding was confirmed by results of the QOL and PS analysis. Indeed, the QOL Index increased with significance at the third interval and the majority of patients were "satisfied", "very satisfied", or "extremely satisfied" at both $\mathrm{T}_{3}$ and $\mathrm{T}_{4}$. We underline these data, as for cancer patients pain therapy should be quickly effective; however, the study design cannot allow extrapolation of exhaustive data. Investigations on specific cancer populations (eg, cancer disease and stage, cancer and other concomitant pharmacological therapies, pain approaches) and focused on detailed outcomes are needed to obtain conclusive data in different settings. In particular, further studies on the topic conducted with a double-blind controlled design will able to overcome the limitations of this observational study, offering in turn more detailed information on drug effectiveness.

\section{Disclosure}

The authors report no conflicts of interest in this work.

\section{References}

1. van den Beuken-van Everdingen MH, Hochstenbach LM, Joosten EA, Tjan-Heijnen VC, Janssen DJ. Update on prevalence of pain in patients with cancer: systematic review and meta-analysis. J Pain Symptom Manage. 2016;51(6):1070-1090. 
2. van Leeuwen M, Husson O, Alberti P, et al. Understanding the quality of life (QOL) issues in survivors of cancer: towards the development of an EORTC QOL cancer survivorship questionnaire. Health Qual Life Outcomes. 2018;16(1):114.

3. Te Boveldt N, Vernooij-Dassen M, Burger N, Ijsseldijk M, Vissers $\mathrm{K}$, Engels Y. Pain and its interference with daily activities in medical oncology outpatients. Pain Physician. 2013;16(4):379-389.

4. Zech DF, Grond S, Lynch J, Hertel D, Lehmann KA. Validation of World Health Organization Guidelines for cancer pain relief: a 10-year prospective study. Pain. 1995;63(1):65-76.

5. Ripamonti CI, Santini D, Maranzano E, Berti M, Roila F. ESMO Guidelines Working Group. Management of cancer pain: ESMO Clinical Practice Guidelines. Ann Oncol. 2012;23(Suppl 7):vii139-154.

6. Caraceni A, Hanks G, Kaasa S, et al. Use of opioid analgesics in the treatment of cancer pain: evidence-based recommendations from the EAPC. Lancet Oncol. 2012;13(2):e58-e68.

7. Chou R, Turner JA, Devine EB, et al. The effectiveness and risks of long-term opioid therapy for chronic pain: a systematic review for a National Institutes of Health Pathways to Prevention Workshop. Ann Intern Med. 2015;162(4):276-286.

8. Benyamin R, Trescot AM, Datta S, et al. Opioid complications and side effects. Pain Physician. 2008;11(2 Suppl):S105-S120.

9. Tzschentke TM, Christoph T, Kögel B, et al. (-)-(1R,2R)-3-(3-dimethylamino1-ethyl-2-methyl-propyl)-phenol hydrochloride (tapentadol $\mathrm{HCl}$ ): a novel mu-opioid receptor agonist/norepinephrine reuptake inhibitor with broadspectrum analgesic properties. JPharmacol Exp Ther. 2007;323(1):265-276.

10. Kress HG. Tapentadol and its two mechanisms of action: is there a new pharmacological class of centrally-acting analgesics on the horizon? Eur J Pain. 2010;14(8):781-783.

11. Meng Z, Yu J, Acuff M, et al. Tolerability of opioid analgesia for chronic pain: a network meta-analysis. Sci Rep. 2017;7(1):1995.

12. Mercadante S, Porzio G, Ferrera P, et al. Tapentadol in cancer pain management: a prospective open-label study. Curr Med Res Opin. 2012;28(11):1775-1779.

13. Kress HG, Koch ED, Kosturski H, et al. Tapentadol prolonged release for managing moderate to severe, chronic malignant tumor-related pain. Pain Physician. 2014;17(4):329-343.

14. Imanaka K, Tominaga Y, Etropolski M, et al. Efficacy and safety of oral tapentadol extended release in Japanese and Korean patients with moderate to severe, chronic malignant tumor-related pain. Curr Med Res Opin. 2013;29(10):1399-1409.

15. Imanaka K, Tominaga Y, Etropolski M, Ohashi H, Hirose K, Matsumura T. Ready conversion of patients with well-controlled, moderate to severe, chronic malignant tumor-related pain on other opioids to tapentadol extended release. Clin Drug Investig. 2014;34(7):501-511.

16. Schikowski A, Krings D, Schwenke K. Tapentadol prolonged release for severe chronic cancer-related pain: effectiveness, tolerability, and influence on quality of life of the patients. J Pain Res. 2015;8:1-8.
17. Wiffen PJ, Derry S, Naessens K, Bell RF. Oral tapentadol for cancer pain. Cochrane Database Syst Rev. 2015;9(9):CD011460.

18. Mercadante S, Porzio G, Adile C, et al. Tapentadol at medium to high doses in patients previously receiving strong opioids for the management of cancer pain. Curr Med Res Opin. 2014;30(10): 2063-2068

19. Carmona-Bayonas A, Jiménez Fonseca P, Virizuela Echaburu J. Tapentadol for cancer pain management: a narrative review. Pain Pract. 2017; 17(8):1075-1088.

20. GRÜNENTHAL ITALIA. Palexia. Foglio Illustrativo: Informazioni Per L'utilizzatore. Available from: https://farmaci.agenziafarmaco.gov.it/aifa/servlet/PdfDownloadServlet?pdfFileName=foo ter_000045_040423_FI.pdf\&retry=0\&sys=m0b113. Accessed July 14, 2018.

21. Mercadante S, Porzio G, Aielli F, et al. Opioid switching from and to tapentadol extended release in cancer patients: conversion ratio with other opioids. Curr Med Res Opin. 2013;29(6):661-666.

22. Oken MM, Creech RH, Tormey DC, et al. Toxicity and response criteria of the Eastern Cooperative Oncology Group. Am J Clin Oncol. 1982;5(6) 649-656.

23. Bouhassira D, Attal N, Alchaar H, et al. Comparison of pain syndromes associated with nervous or somatic lesions and development of a new neuropathic pain diagnostic questionnaire (DN4). Pain. 2005;114(1-2): 29-36.

24. Mercadante S, Dardanoni G, Salvaggio L, Armata MG, Agnello A Monitoring of opioid therapy in advanced cancer pain patients. J Pain Symptom Manage. 1997;13(4):204-212.

25. Addington-Hall JM, MacDonald LD, Anderson HR. Can the Spitzer Quality of Life Index help to reduce prognostic uncertainty in terminal care? Br J Cancer. 1990;62(4):695-699.

26. Bennett MI, Rayment C, Hjermstad M, Aass N, Caraceni A, Kaasa S. Prevalence and aetiology of neuropathic pain in cancer patients: a systematic review. Pain. 2012;153(2):359-365.

27. Sugiyama Y, Kataoka T, TasakiY, et al. Efficacy of tapentadol for first-line opioid-resistant neuropathic pain in Japan. Jpn J Clin Oncol. 2018;48(4): 362-366.

28. Falk S, Patel R, Heegaard A, Mercadante S, Dickenson AH. Spinal neuronal correlates of tapentadol analgesia in cancer pain: a backtranslational approach. Eur J Pain. 2015;19(2):152-158.

29. Ono H, Nakamura A, Kanbara T, et al. Effect of the norepinephrine transporter (NET) inhibition on $\mu$-opioid receptor (MOR)-induced antinociception in a bone cancer pain model. J Pharmacol Sci. 2014;125(3): 264-273.

30. Mercadante S, Bruera E. Opioid switching: a systematic and critical review. Cancer Treat Rev. 2006;32(4):304-315.

31. Cascella M, Cuomo A, Viscardi D. Pharmacological approaches. In: Cascella M, Cuomo A, Viscardi D, editors. Features and Management of the Pelvic Cancer Pain. Verlag: Springer; 2016:73-93.

\section{Journal of Pain Research}

\section{Publish your work in this journal}

The Journal of Pain Research is an international, peer reviewed, open access, online journal that welcomes laboratory and clinical findings in the fields of pain research and the prevention and management of pain. Original research, reviews, symposium reports, hypothesis formation and commentaries are all considered for publication.
The manuscript management system is completely online and includes a very quick and fair peer-review system, which is all easy to use. Visit http://www.dovepress.com/testimonials.php to read real quotes from published authors. 